\title{
Curcumin-PVP Loaded Electrospun Membranes with Conferred Antibacterial and Antitumoral Activities
}

\author{
Gyuldzhan Yakub $^{1}$, Antoniya Toncheva ${ }^{1}$, Veselin Kussovski ${ }^{2}$, Reneta Toshkova ${ }^{3}$, Ani Georgieva ${ }^{3}$, \\ Elena Nikolova $^{3}$, Nevena Manolova ${ }^{1 *}$, and Iliya Rashkov ${ }^{1}$ \\ ${ }^{1}$ Laboratory of Bioactive Polymers, Institute of Polymers, Bulgarian Academy of Sciences, Sofia 1113, Bulgaria \\ ${ }^{2}$ Institute of Microbiology, Bulgarian Academy of Sciences, Sofia 1113, Bulgaria \\ ${ }^{3}$ Institute of Experimental Morphology, Pathology and Anthropology with Museum, Bulgarian Academy of Sciences, \\ Sofia 1113, Bulgaria \\ (Received May 8, 2019; Revised July 18, 2019; Accepted July 19, 2019)
}

\begin{abstract}
Electrospun membranes containing curcumin were prepared from poly(L-co-D,L-lactic) acid and polyvinylpyrrolidone. The effect of curcumin concentration on the solution viscosity and the morphology of fiber was studied. Curcumin solubility in aqueous solutions was enhanced by the formation of curcumin/polyvinylpyrrolidone watersoluble complex. Curcumin physico-chemical and therapeutic properties within the membranes were preserved upon UV-Vis light irradiation, as a part of the membranes sterilization. The biomaterials showed antibacterial activity against pathogenic microorganisms such as Staphylococcus aureus and Candida albicans. In-vitro experiments against HeLa and Graffi tumor cells and white blood cells (peritoneal macrophages and spleen lymphocytes) revealed potential biomedical application of the membranes.
\end{abstract}

Keywords: Nanomaterials, Electrospinning, Polylactic acid, Curcumin, Antibacterial and antitumoral activity

\section{Introduction}

During the last years the biocompatible polymers gained significant scientific interest with a focus on the production of new generation polymer devices with direct application in the medical and pharmaceutical field. A promising approach in their effective nanostructuration has turned out to be the use of electrospinning. Some of the major advantages of the fibrous mats are the relative ease in their fabrication, their large specific surface area and lightness, as well as the possibility to confer them desired properties through the incorporation of bioactive substances, nanoparticles or oligomers. Therefore, the developed active materials can be used in the manufacturing process of drug delivery systems, antibacterial and anesthetic wound dressing, scaffolds for tissue engineering or for open wounds treatment [1-3].

Curcumin (Curc), also known as yellow gold, is a naturally occurring substance with polyphenol structure usually extracted from Curcuma Longa plant having a rich palette of therapeutic properties (antioxidant, anti-inflammatory, antibacterial, antifungal, etc.) [4]. However, its low watersolubility and relative chemical instability (alkaline $\mathrm{pH}$ ) significantly reduce its bioavailability and prevents living organisms from easily absorbing it $[5,6]$. In the scientific literature multiple solutions were proposed on tackling this issue by incorporating Curc in liposomes, micelles, emulsions and nanoparticles [7]. Another promising approach to improve its water-solubility and long-term local delivery is Curc loading in micro- and nanofibrous materials. They are characterized with a significant level of loading capacity and

*Corresponding author: manolova@polymer.bas.bg encapsulation efficiency. It was demonstrated that the antioxidant water-solubility can be improved by choosing appropriate fibers polymer matrix. Following this idea electrospun mats were successfully produced from: poly(Lco-D,L-lactic)acid/poly(ethylene glycol) blends or polylactic acid/polyvinylpyrrolidone (PLA/PVP) blends [8,9], polylactic acid/cellulose acetate [10] or cellulose acetate/PVP $[11,12]$, poly(vinyl alcohol) containing $\beta$-cyclodextrin [13], poly( $\varepsilon$ caprolactone)/tragacanth [14] or mats made out of chitosan/ polylactic acid blends [15]. An interesting covalent binding between the polymer chains [(meth)acrylate family polymers] and Curc was also described [16].

As part of the latest trends in modern medicine, the fabrication of effective and less toxic biomaterials with conferred antitumoral properties is largely discussed. Recently it was demonstrated that the electrospun mats can undergo significant progress in this field [17]. Well known for its biocompatibility and relatively low toxicity, Curc represents a good candidate for replacement of common antitumoral agents and has already been loaded in polylactic acid fibers with a proven cytotoxic activity against C6 glioma cells in mice [18], in polylactic acid/cellulose acetate and polylactic acid/silk fibroin with an impact on breast cancer cells [10], in PVP for melanoma cancerous cells studies [19] in poly(lactic-co-glycolic acid) in treatment tests with human carcinoma [20] and in amphiphilic block copolymer of poly( $\varepsilon$-caprolactone)-poly(ethylene glycol)poly( $\varepsilon$-caprolactone) fibers for post-operative interventions in rats with brain tumors [21]. However, the design of these polymer materials is not always offering the possibility to enhance the water-solubility of Curc, and at the same time preserve its chemical structure and therapeutic properties for 
long-term release profile.

The aim of this study is to produce active and stable upon UV-Vis irradiation electrospun materials with conferred antibacterial and antitumor properties through the incorporation of Curc into biocompatible polymer matrix made of PLA and PVP. The morphology of the fibers is evaluated by scanning electronic and fluorescent microscopy and the fibers mean diameter is discussed in a direct correlation with the spinning solution dynamic viscosity. The complex formation, based on hydrogen bonds between Curc and the polymer chains, is studied in detail and its impact on the preservation of the properties of the active substance is evaluated upon UV-Vis irradiation. Special attention is paid to Curc release profile and this in relation with the polymer matrix composition. The antibacterial properties of the PLA/ PVP/Curc membranes are studied against pathogen microorganisms such as Staphylococcus aureus ( $S$. aureus) and Candida albicans ( $C$. albicans). In addition, the cytotoxic effect of PVP/Curc and PLA(/PVP)/Curc fibers is investigated performing experiments with tumor HeLa and Graffi cell lines. The immune response of the membranes is monitored after contact with white blood cells (peritoneal macrophages and spleen lymphocytes).

\section{Experimental}

\section{Materials}

The membranes were produced from poly(L-co-D,L-lactic acid) [PLA, Boehringer Ingelheim Chemicals (Germany)] with $\mathrm{L} / \mathrm{D}, \mathrm{L}=70 / 30, \overline{\mathrm{M}}_{\mathrm{n}}=78500 \mathrm{~g} / \mathrm{mol}, \overline{\mathrm{M}}_{\mathrm{w}}=165850 \mathrm{~g} / \mathrm{mol}$ and $\left.\overline{\mathrm{M}}_{\mathrm{w}} / \overline{\mathrm{M}}_{\mathrm{n}}=2.1\right]$, polyvinylpyrrolidone (PVP, Fluka) with $\overline{\mathrm{M}}_{\mathrm{r}}=360000 \mathrm{~g} / \mathrm{mol}$ and curcumin (Curc) with $\mathrm{M}=368,38 \mathrm{~g} /$ mol (Merck). Dichloromethane (DCM), dimethyl sulfoxide (DMSO) and ethanol (EtOH) were provided by Merck and Fluka, respectively. For the microbiology tests Staphylococcus aureus 509 and Candida albicans 74 from National bank for industrial microorganisms and cell cultures were used. Cervical cancer HeLa cells line were cultivated in a DMEM media (Gibco, Austria) with $10 \%$ fetal bovine serum (Gibco, Austria), $100 \mathrm{U} / \mathrm{ml}$ penicillin and $0.1 \mathrm{mg} / \mathrm{m} l$ streptomycin while using $75 \mathrm{~cm}^{3}$ tissue plastic flasks. Trypsin- ethylenediaminetetraacetic acid, penicillin and streptomycin were acquired by FlowLab, Australia. For the culture cell tests, DMSO and 3-(4,5-dimethylthiazol-2-yl)2,5-diphenyltetrazolium bromide (MTT) were purchased from Sigma Aldrich, Germany.

\section{Preparation of the Fibrous Materials}

PVP/Curc fibers were produced from spinning solutions obtained after mixing a solution containing PVP (0.49 g) dissolved in $4 \mathrm{ml} \mathrm{EtOH}$ and a solution containing different amount of Curc (10, 15 and $25 \mathrm{wt} \%$ with respect to the final polymer weight) dissolved in $4.2 \mathrm{ml}$ of EtOH. PLA/PVP/ Curc fibers were obtained after mixing a solution of PLA
$(0.35 \mathrm{~g})$ dissolved in $5.2 \mathrm{~m} l$ DCM and a solution of PVP $(0.35 \mathrm{~g})$ dissolved in $2.1 \mathrm{~m} l$ DMSO. Then the respective amount of Curc was added to the final solution.

The electrospinning setup consisted of a high voltage power supply (up to $30 \mathrm{kV}$ ), a pump for controlled spinning solution delivering (NE-300 Just Infusion Syringe Pump (New Era Pump Systems Inc., USA)), a syringe equipped with a metal needle (gauge of 20 GX11/2") connected to the positively charged electrode and a grounded rotating aluminum collector (diameter of $5.6 \mathrm{~cm}$ ). In the case of the PVP/Curc fibers the working conditions were: spinning solution feeding rate of $3 \mathrm{ml} / \mathrm{h}$, applied voltage of $25 \mathrm{kV}$, tip to the collector distance of $10 \mathrm{~cm}$ and collector rotation speed of $2000 \mathrm{rpm}$. For PLA/PVP and PLA/PVP/Curc membranes these parameters were: $3 \mathrm{ml} / \mathrm{h}, 29 \mathrm{kV}, 20 \mathrm{~cm}$ and $2000 \mathrm{rpm}$, respectively.

\section{Characteristics of the Spinning Solutions and the Fibrous Materials}

Detailed morphological analyses of the membranes were done using scanning electron microscope (SEM Jeol JSM5510 and Philips SEM 515) after vacuum gold-coating the samples. The mean fiber diameter was determined using ImageJ software, while measuring the diameter of at least 50 individual fibers. Fluorescent micrographs were obtained operating with fluorescent microscope (NU-2; Carl Zeiss Jena, Germany) at maximum wavelength of excitation $\left(\lambda_{\mathrm{ex}}\right)$ of $420 \mathrm{~nm}$ and maximum wavelength of emission $\left(\lambda_{\mathrm{em}}\right)$ of $470 \mathrm{~nm}$. The dynamic spinning solution viscosity was measured at temperature of $25^{\circ} \mathrm{C}$ with Brookfield viscometer and Brookfield TC-102 thermostat.

Infrared spectra (Fourier transformation, FT-IR) of the membranes were done with IRAffinity-1 spectrophotometer (Shimadzu Co., Japan) supplied with a MIRacle ATR add-on (crystal diamond; depth penetration of IR beam of $2 \mu \mathrm{m}$ in the range from 4000 to $600 \mathrm{~cm}^{-1}$ and resolution of $4 \mathrm{~cm}^{-1}$; PIKE Technologies, USA). All data were corrected for $\mathrm{H}_{2} \mathrm{O}$ and $\mathrm{CO}_{2}$ (IRsolution software). In order to study the materials degree of crystallinity, X-ray diffraction analyses (XRD; D8 Bruker Advance dust diffractometer) were done at room temperature with filtered $\mathrm{Cu} \mathrm{K}$ source of radiation and a luminescent detector in the $2 \theta$ range from $0^{\circ}$ to $80^{\circ}$, step of $0.02^{\circ}$ and a countdown timer of $1 \mathrm{sec} / \mathrm{step}$. The fibrous materials thermal properties were studied performing thermogravimetric analysis (TGA; TGA Q5000) by heating the samples from 0 to $1000{ }^{\circ} \mathrm{C}$ and differentially scanning calorimetry (DSC; DSC Perkin Elmer DSC 8500) in the range from 0 to $250{ }^{\circ} \mathrm{C}$ while applying heating/cooling speed of $10^{\circ} \mathrm{C} / \mathrm{min}$ under nitrogen atmosphere. Curc content in the fibrous materials was determined spectrophotometrically [DU 800 UV, Beckman Coulter spectrophotometer] based on calibration curves at wavelength of $426 \mathrm{~nm}$ [EtOH was used as a solvent for PVP/Curc membranes and DCM/ DMSO (75/25 wt/wt) for (PLA/)PVP/Curc fibers]. 
The impact of UV-Vis light irradiation (light range from 260 to $600 \mathrm{~nm}$ and source to sample distance of $43 \mathrm{~cm}$ ) on Curc physico-chemical properties loaded in the fibers was studied after 30,60 and $120 \mathrm{~min}$ or irradiation time with respective radiation dosage of 261,522 and $1044 \mathrm{~J} / \mathrm{cm}^{2}$. After this step, the membranes were dissolved in $\mathrm{EtOH}$ and the solution absorption was registered at wavelength of $426 \mathrm{~nm}$. The Curc residual amount within the membranes presented an average value from three separate experimental setups, applying equation (1):

$$
\text { Curc residual amount }(\%)=\left(C_{i r r} / C_{0}\right) \times 100
$$

where $C_{i r r}$ is the content of Curc in the membranes after 30 , 60 or $120 \mathrm{~min}$ of UV-Vis irradiation and $C_{0}$ is the Curc content in the relative non-irradiated membrane.

The surface chemical composition of the membranes was studied by X-ray photoelectron spectroscopy (XPS). The measurements were taken in a vacuum UHV camera with an ESCALAB-Mkll (VG Scientific) electronic spectrometer applying monochrome $\mathrm{Mg} \mathrm{K}_{\alpha}$ source irradiation with final resolution of $1 \mathrm{eV}$. An energy calibration was performed, using as reference the binding energy for $\mathrm{C}-\mathrm{H}$ hydrogen bonds at $285 \mathrm{eV}\left(\mathrm{C}_{1 \mathrm{~s}}\right)$.

Hydrophobic/hydrophilic surface behavior of the membranes was determined after measuring the water contact angle (Easy Drop DSA20E KRUSS GmbH apparatus). To find out the correct values, $10 \mu l$ drops of deionized water were deposited on the membranes surface at room temperature after measuring at least 10 independent drop profiles for each sample.

The morphological changes in the PLA/PVP and PLA/ $\mathrm{PVP} /$ Curc membranes after $24 \mathrm{~h}$ immersion in distilled water and subsequent freeze-drying were evaluated with SEM. The weight loss was obtained by measuring the weight difference of the sample before their immersion and after their lyophilization.

\section{Curc Release Profile}

The release profile of the bioactive substance from the fibers was studied at a temperature of $37{ }^{\circ} \mathrm{C}$, in an acetate buffer/PVP=90/10 v/v (PVP with $\overline{\mathrm{M}}_{\mathrm{r}}$ of $360000 \mathrm{~g} / \mathrm{mol}$; buffer ionic strength of 0.1 ) as release medium with $\mathrm{pH}$ of 5.5. PLA/Curc 30 and $\mathrm{PLA}_{50} / \mathrm{PVP}_{50} / \mathrm{Curc}_{15}$ membranes were immerged in $100 \mathrm{~m} l$ of the prepared buffer, while applying a stirring of $200 \mathrm{rpm}$. At specific intervals of time, aliquots $(2 \mathrm{ml})$ were withdrawn and the solution absorption at a wavelength of $439 \mathrm{~nm}$ was registered. Afterwards, the withdrawn volumes were replaced with fresh buffer solution. The amount of the diffused from the membranes Curc was calculated using calibration curve and the results were presented as average values of three separate trials per membrane. The initial Curc amount in the PLA/Curc 30 and $\mathrm{PLA}_{50} / \mathrm{PVP}_{50} / \mathrm{Curc}_{15}$ membranes was determined after dissolving the fibers in appropriate volume of DCM/ $\mathrm{DMSO}=75 / 25(\mathrm{wt} / \mathrm{wt})$ and measuring the solution absorption at wavelength of $426 \mathrm{~nm}$.

\section{Microbiological Assays}

The minimum inhibitory concentration (MIC) of Curc was studied against two pathogen microorganisms: Grampositive bacteria $S$. aureus and the fungus $C$. albicans at bacterial suspensions concentration of $10^{6} \mathrm{cells} / \mathrm{ml}$. The microbiological tests with the PVP/Curc and PLA/PVP/Curc membranes were performed upon irradiation $(420 \mathrm{~nm})$ or in a dark environment following previously described methodology [8].

\section{Tumor Cell Viability}

HeLa cancer cells were first cultured in DMEM by adding $10 \% \mathrm{FBS}, 100 \mathrm{U} / \mathrm{m} l$ penicillin, and $0.1 \mathrm{mg} / \mathrm{m} l$ streptomycin and placed in $\mathrm{CO}_{2}$ incubator $\left(37^{\circ} \mathrm{C}, 90 \%\right.$ humidity and $5 \%$ $\mathrm{CO}_{2}$ ). At 80 to $90 \%$ of cell confluence, the cell culture was treated with $0.25 \%$ trypsin-EDTA solution and then counted using hemocytometer. As a next step, 96 well culture plates were used with cells concentration of $2 \times 10^{4}$ cell/well. After $24 \mathrm{~h}$ of incubation the culture medium was removed and the HeLa cells were put in presence of PVP, $\mathrm{PLA}_{50} / \mathrm{PVP}_{50}$, $\mathrm{PLA}_{50} / \mathrm{PVP}_{50} / \mathrm{Curc}_{15}$ and $\mathrm{PVP} / \mathrm{Curc}_{15}$ membranes for 24, 48 and $72 \mathrm{~h}$ of contact. In the case of the Graffi tumor cells, RPMI-1640 culture media was used (enriched with $10 \%$ fetal bovine serum, $100 \mathrm{U} / \mathrm{m} l$ penicillin and $0.1 \mathrm{mg} / \mathrm{m} l$ streptomycin) and cell $\mathrm{CO}_{2}$ incubator at $37^{\circ} \mathrm{C}, 90 \%$ humidity and $5 \% \mathrm{CO}_{2}$. The Graffi cell culture was treated with Trypsin-EDTA, counted with Trypan blue and split in 96 well culture plates $\left(2 \times 10^{4}\right.$ cells/well $)$. After $24 \mathrm{~h}$ of incubation, the culture medium was removed and the cells were put in contact with PVP, $\mathrm{PLA}_{50} / \mathrm{PVP}_{50}, \mathrm{PLA}_{50} / \mathrm{PVP}_{50} /$ Curc $_{15}$ and PVP/Curc 15 membranes for 24, 48 and $72 \mathrm{~h}$ of contact. In both HeLa and Graffi cells studies, Curc was used as a positive control. After culturing, the cells were washed out twice with PBS (pH 7.4) and additionally incubated with $100 \mu l$ MTT solution (Sigma Chemical Co.) for $3 \mathrm{~h}$ at $37{ }^{\circ} \mathrm{C}$. Then the supernatants were took out and $100 \mu l$ lysing solution (DMSO/ethanol=1:1) was added in order to dissolve any presence of formazan crystals. The cells viability was evaluated applying MTT test [22] using ELISA plate reader (TECAN, SunriseTM, Grödig/Salzburg, Austria). The cell vitality was expressed as percentage based on equation (2):

$$
\text { Cell viability }(\%)=\left(\mathrm{OD}_{570 \mathrm{exp}} / \mathrm{OD}_{570 \text { control }}\right) \times 100
$$

where $\mathrm{OD}_{570 \text { exp }}$ is the absorption value $(\lambda=570 \mathrm{~nm})$ for the cell culture at given time of contact with the membranes or after the addition of Curc, and $\mathrm{OD}_{570 \text { control }}$ is the absorption value $(\lambda=570 \mathrm{~nm})$ for the control cell culture. 


\section{Proliferation Cell Tests of Murine Spleen Lymphocytes and Peritoneal Macrophages}

For this study male and female healthy mice (6 to 8 weeks old, weigh of 18-20 g) were purchased from a certified laboratory (SBALHZ-Oncology, Sofia, Bulgaria). All animals were grown at standard temperature $\left(20 \pm 5{ }^{\circ} \mathrm{C}\right)$, were fed dietary standard pellets and had free access to water. They were carried in accordance with the requirements of the recommended institutionally recommended guidelines. The animal experiments were conducted in accordance with the requirements of the Animal Ethics Committee. After scarifying the animals, peritoneal cells were isolated by a carful procedure of peritoneal cavity washing with $10 \mathrm{~m} l$ of sterile cold PBS ( $\mathrm{pH}$ 7.2). As next step the cell suspensions were washed twice with RPMI 1640 medium containing $10 \%$ fetal bovine serum. In order to determine the cell viability (in contact with the membranes and presence of Curc) was evaluated with a hematocytometer, while using tryptone blue dye as cell viability test using 96-well plate $\left(1 \times 10^{6}\right.$ cells $\left./ \mathrm{m} l\right)$ with RPMI-1640 containing $10 \%$ fetal bovine serum.

In the case of the spleen cells study, the mice were sacrificed by cervical dislocation. The spleens organs were aseptically removed, placed and cut in small pieces in $60 \mathrm{~m} l$ tissue culture vessels containing $5 \mathrm{~m} l$ culture medium. After this step, the tissue fragments were filtered through nylon tissue and the cell suspensions were placed on a Ficoll pack density gradient and centrifuged at $2200 \mathrm{rpm}$ for $20 \mathrm{~min}$ at $4{ }^{\circ} \mathrm{C}$. The collected lymphocyte-enriched fraction was rinsed twice with RPMI-1640 medium containing $10 \%$ fetal bovine serum, centrifuged and resuspended in the same medium at a concentration of $1 \times 10^{6}$ cells $/ \mathrm{m} l$. The number of cells and their viability (in contact with the membranes and in the presence of Curc) were determined by tests with tryptone blue dye.

\section{Results and Discussion}

In the present contribution, biocompatible PVP and PLAbased membranes loaded with the natural product Curc were produced by electrospinning while conferring desired morphological characteristics to the fibers. The electrospinning of the PVP and PVP/Curc (Curc content of 10 or $15 \mathrm{wt} \%$ ) spinning solutions led to the deposition of defect free cyclical fibers (Figure 1(a) and (b)). It was found that the fibers' morphology and their specific surface area can be subject of additional design by increasing the content of the natural substance and this is achievable without changing the electrospinning process parameters. For example, in the case of PVP/Curc ${ }_{20}$ and $\mathrm{PVP} / \mathrm{Curc}_{25}$ membranes, the fibers were ribbon-like shaped (Figure 1(c)). In the literature, such structures are described as micro- and nanoribbons and can often be the result of electrospinning of concentrated solutions at conditions of high degree of humidity at short

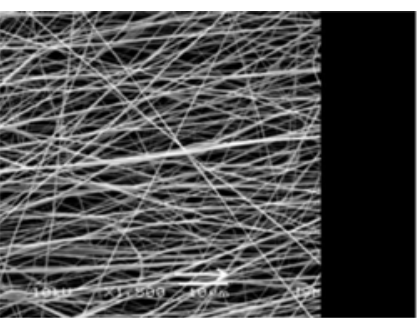

(a)

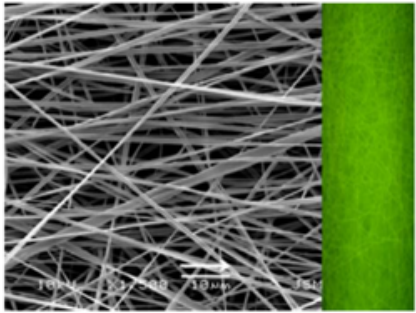

(c)

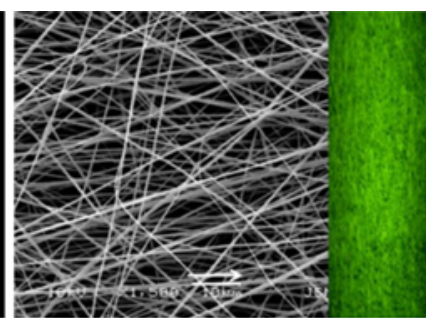

(b)

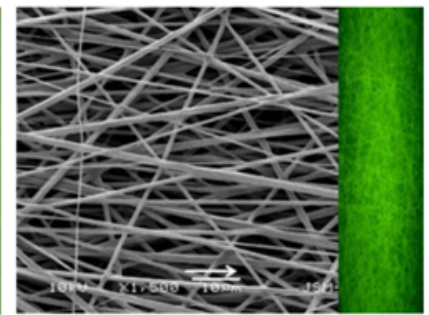

(d)
Figure 1. SEM micrographs of membranes of PVP (a), PVP/ Curc $_{15}$ (b), PVP/Curc 25 (c) and $\mathrm{PLA}_{50} / \mathrm{PVP}_{50} / \mathrm{Curc}_{15}$ (d). The collector rotation direction is indicated with an arrow. SEM micrographs magnification: $\times 1500$; fluorescent microscopy images.

Table 1. Average values of dynamic viscosity of the spinning solutions and average fiber diameter

\begin{tabular}{lcc}
\hline \multicolumn{1}{c}{$\begin{array}{c}\text { Membrane } \\
\text { composition }\end{array}$} & $\begin{array}{c}\text { Dynamic viscosity } \\
(\mathrm{cP})\end{array}$ & $\begin{array}{c}\text { Mean diameter } \\
(\mathrm{nm})\end{array}$ \\
\hline $\mathrm{PVP}$ & $50.4 \pm 0.2$ & $502 \pm 13$ \\
$\mathrm{PVP} / \mathrm{Curc}_{10}$ & $57.3 \pm 0.1$ & $560 \pm 126$ \\
$\mathrm{PVP} / \mathrm{Curc}_{15}$ & $57.5 \pm 0.1$ & $692 \pm 125$ \\
$\mathrm{PVP} / \mathrm{Curc}_{20}$ & $56.0 \pm 0.1$ & $764 \pm 164$ \\
$\mathrm{PVP} / \mathrm{Curc}_{25}$ & $58.0 \pm 0.1$ & $1073 \pm 177$ \\
$\mathrm{PLA}_{50} / \mathrm{PVP}_{50}$ & $108.8 \pm 1.6$ & $939 \pm 184$ \\
$\mathrm{PLA}_{50} / \mathrm{PVP}_{50} / \mathrm{Curc}_{15}$ & $119.6 \pm 2.3$ & $1064 \pm 191$ \\
\hline
\end{tabular}

capillary to collector distance $[23,24]$. Their unique anisotropic structure (high aspect ratio values) could be of particular interest for the preparation of membranes with specific properties (optical, physico-mechanical) or systems with desired drug release rate. In this study, we observed a change in the fibers morphology from cylindrical to ribbon-like (Curc content higher than $15 \mathrm{wt} \%$ ). Since hydrogen bond complex formation proceeds between PVP and Curc [25], the spinning solution dynamic viscosity changes (Table 1) and has an effect on the fiber morphology.

From the already published data it is known that the electrospinning of concentrated polymer solutions leads to the formation of fibers with higher diameter values, largely because of the higher degree of the polymer chains entanglements [26]. Here, we demonstrated that another factor could be the degree of complex formation between the spinning solution components: increase in diameter with the 
increase of the antioxidant amount (Table 1). It was found that PVP fibers had a diameter of $502 \pm 13 \mathrm{~nm}$, while the cross section of $\mathrm{PVP} / \mathrm{Curc}_{25}$ was almost twice as large $(1073 \pm 177 \mathrm{~nm})$ at a viscosity values of $58.0 \pm 0.1 \mathrm{cP}$. As far as the $\mathrm{PLA}_{50} / \mathrm{PVP}_{50}$ membranes, the fibers were cylindrical with an average diameter of $939 \pm 184 \mathrm{~nm}$ with presence of local inter-fiber adhesion zones (Table 1). These instabilities of the electrospinning process were stabilized with the addition of the hydrophobic Curc to the $\mathrm{PLA}_{50} / \mathrm{PVP}_{50}$ solution due to hydrogen bonds complex formation between Curc and the macromolecules. Such improvement of the fibers morphology (from defects interconnected fibers to well defined individual fibers), after adding Curc to electrospinning spinning solutions was recently discussed [27]. In addition to this, $\mathrm{PLA}_{50} / \mathrm{PVP}_{50} / \mathrm{Curc}_{15}$ fibers were obtained with diameter of $1064 \pm 191 \mathrm{~nm}$ at viscosity values of $119.6 \pm 2.3 \mathrm{cP}$. Taking advantage of the polyphenol fluorescent properties, it was possible to observe its distribution along the fibers length using fluorescence optical microscopy (Figure 1).

A confirmation for the complex formation between the bioactive substance and the polymer chains in the electrospun materials was obtained by IR-spectroscopy (Supporting information, Figure S1). The main changes in the spectra were related to: (i) displacement of the

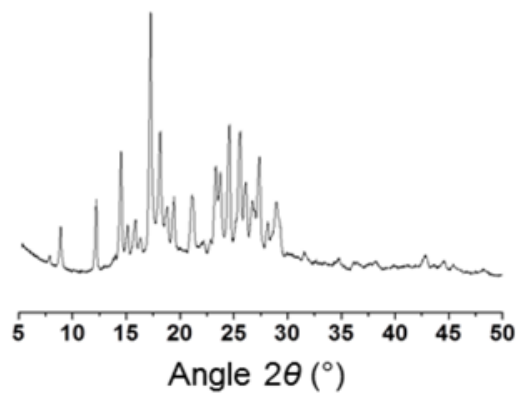

(a)

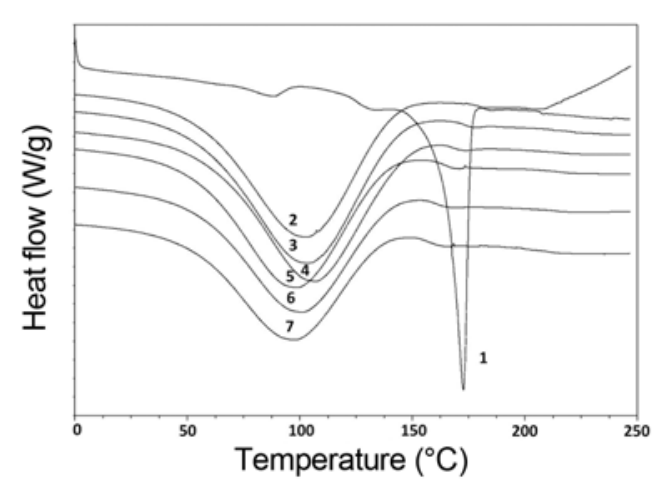

(c) characteristic for $\mathrm{C}=\mathrm{O}$ band in the structure of $\mathrm{PVP}$ from $1651 \mathrm{~cm}^{-1}$ to $1657 \mathrm{~cm}^{-1}$ (PVP/Curc membranes), (ii) displacement of the characteristic for PLA $\mathrm{C}=\mathrm{O}$ band from 1749 to $1755 \mathrm{~cm}^{-1}$ (PVP/PLA/Curc membranes) and (iii) disappearance of the characteristic band for Curc at 3508 $\mathrm{cm}^{-1}$ (OH group from the Curc phenolic group) in the case of PVP/Curc and PLA/PVP/Curc membranes. This data were in agreement with the published results relative to the intermolecular interaction between PVP and Curc or PVP and D,L-units of the poly(D,L-lactic acid) [28,29].

The release profile of a given bioactive substance is directly depending on its physical state (crystal or amorphous) in the membrane, its specific location in the fibers (on their surface or in their volume), its interaction with the polymer chains (complex formation) and its physico-chemical stability in the final polymer material. It only follows, that it was of a particular interest to find out if Curc was in an amorphous or crystal state in the electrospun (PLA)PVP/ Curc fibers. It is known that Curc diffraction peaks can be found at $2 \theta$ values of 14.5, 17.2, 17.7, 24.6, 25.2 ${ }^{\circ}$ [30] (Figure 2(a)). It is interesting to note that although polylactic acid of $L / D, L=70 / 30$ composition is amorphous [31,32], presence of crystalline phase for PLA was detected at $2 \theta$ value of $22.0^{\circ}$. This may be attributed to strain-induced changes in the crystal morphology, similarly to reports in

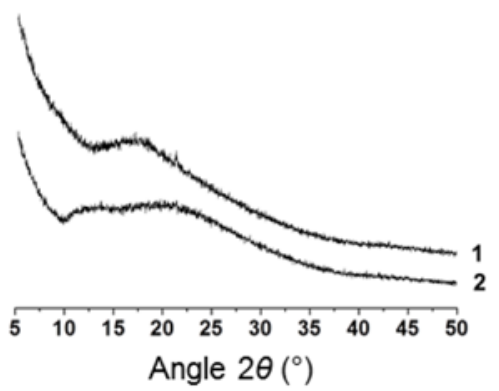

(b)

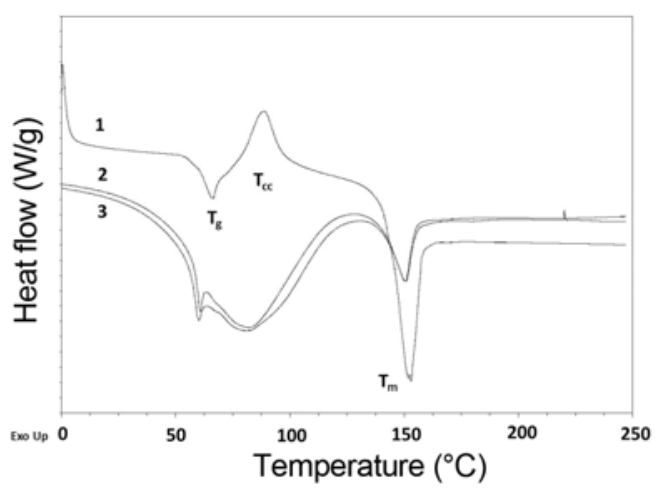

(d)

Figure 2. X-ray diffraction spectra of Curc (crystalline state) (a) and $\mathrm{PLA}_{50} / \mathrm{PVP}_{50} / \mathrm{Curc}_{15}$ (1) and PVP/Curc 25 (2) membranes (b). DSC thermograms of Curc (1) and membranes of PVP (2), PVP/Curc 5 (3), PVP/Curc 10 (4), PVP/Curc ${ }_{15}$ (5), PVP/Curc 20 (6) and PVP/Curc 25 (7) presented in (c) and membranes of PLA (1), $\mathrm{PLA}_{50} / \mathrm{PVP}_{50}$ (2) and $\mathrm{PLA}_{50} / \mathrm{PVP}_{50} / \mathrm{Curc}_{15}$ (3) presented in (d). 
literature [33]. For PVP/Curc 25 and $\mathrm{PLA}_{50} / \mathrm{PVP}_{50} / \mathrm{Curc}_{15}$ membranes the peaks for Curc were not observed, revealing its amorphous state. We assumed that this was a result of the complex formation between the polyphenol and the macromolecules preventing the formation of Curc crystal structures (absence of Curc melting point; DSC thermograms presented in Figure 2(c) and (d)). These overall results were a prerequisite for a gradual release of Curc from the fibrous materials by avoiding burst release for continuous therapeutic effect (see Release profile section). Additionally, after 9month storage period of the nanofibers (temperature of $25^{\circ} \mathrm{C}$, dark environment), the polyphenol compound remained in amorphous state.

Up to this date, the question of Curc chemical stability and storage once incorporated in the micro- or nanofibers is still barely studied in the literature. It is established that Curc could be subject to photo-destruction when light-irradiated (UV-Vis spectrum range) in solution or in its crystal dry state. As a result, several degradation products can be obtained (vanillin, vanillic acid, ferulic acid and other) thus losing the polyphenol therapeutic properties [34]. This limits in higher degree the applications of final Curc-loaded devices as drug delivery systems, packaging materials with antibacterial properties, wound healings and others. In our study, the fresh obtained membranes were irradiated with UV-Vis light source $(260-600 \mathrm{~nm})$ for 30, 60 and $120 \mathrm{~min}$. After 120 min of light exposure, the greatest residual amount of Curc was observed for the PVP/Curc membrane (85\%) and in less in the fibers containing the polyester (around $70 \%$ for PLA/Curc and $75 \%$ for $\mathrm{PLA}_{70} / \mathrm{PVP}_{30} /$ Curc Figure $3)$. This was an indication that the presence of the hydrophilic polymer favors the preservation of Curc chemical structure upon intensive irradiation. In addition, the amount of Curc after 9 months of storage (dark environment at $25{ }^{\circ} \mathrm{C}$ ) did not change significantly remaining $95 \%$ of its initial content in the fibers. On the basis of the obtained results, we assumed that the electrospun materials could be suitable for biomedical application and be a subject of surface sterilization upon UV-irradiation without losing the

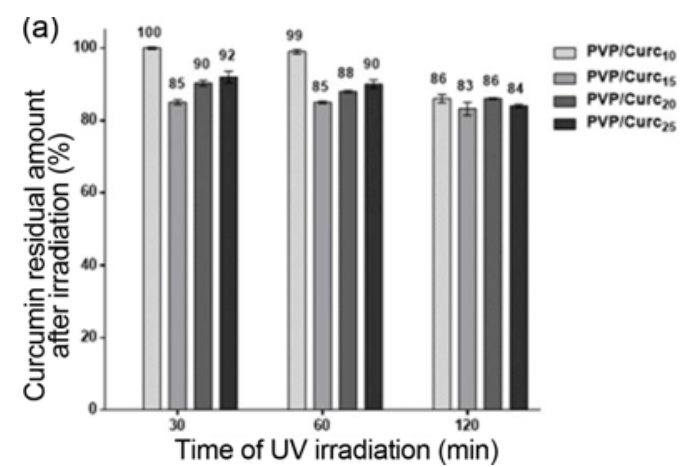

physico-chemical and therapeutic properties of the incorporated bioactive substance.

The deconvoluted $\mathrm{C} 1 \mathrm{~s}$ spectra of the fibrous materials were also studied in detail (membranes of PVP(/Curc) and PLA/PVP(/Curc) (Supporting information, Figure S2). In all cases three peaks at $288 \mathrm{eV}, 286 \mathrm{eV}$ and $285 \mathrm{eV}$ were observed, characteristic for carbon atom engaged in chemical $-\underline{\mathrm{C}}=\mathrm{O}, \underline{\mathrm{C}}-\mathrm{O}, \underline{\mathrm{C}}-\mathrm{N}$ and $\underline{\mathrm{C}}-\mathrm{H} / \underline{\mathrm{C}}-\mathrm{C}$ bonds. For all the materials, the largest surface area carries the peak at $285 \mathrm{eV}$, which is an evident proof of the fibers surface enrichment with $\mathrm{C}-\mathrm{H} / \mathrm{C}-\mathrm{C}$ chemical bonds. For the PVP membrane, the experimentally derived relationship for the peaks surface was $[\underline{\mathrm{C}}-\mathrm{H} / \underline{\mathrm{C}}-\mathrm{C}] /[\underline{\mathrm{C}}-\mathrm{O}, \underline{\mathrm{C}}-\mathrm{N}] /[\underline{\mathrm{C}}=\mathrm{O}]=48 / 34 / 18$. In this case, we concluded that the PVP macromolecules were oriented in such a way upon the fibers production that the carbonyl group and the nitrogen atom were located with priority in volume of the fibers, while the $-\left(\mathrm{CH}_{2}\right)_{3}$ - skeleton was placed on their surface. This result is in line with the data in the literature concerning a heightened affinity to air environments of hydrophobic fragments in different type of surfaces [35]. Additional surface hydrophobization was noticed in the PVP fibers after the Curc addition. The peaks surface relation for the $\mathrm{PVP} / \mathrm{Curc}_{10}$ membrane was $[\underline{\mathrm{C}}-\mathrm{H} / \underline{\mathrm{C}}-\mathrm{C}] /[\underline{\mathrm{C}}-\mathrm{O}, \underline{\mathrm{C}}-\mathrm{N}] /$ $[\underline{\mathrm{C}}=\mathrm{O}]=65 / 24 / 11$, and $62 / 26 / 12$ for $\mathrm{PVP} / \mathrm{Curc}_{25}$. This illustrated a tendency towards achieving new macromolecular stable state in presence of the antioxidant hydrophobic molecule: further migration of the polymer molecule fragments to the volume of the fibers. Similar decreases of the surface area for $\underline{\mathrm{C}}-\mathrm{H} / \underline{\mathrm{C}}-\mathrm{C}$ and $\underline{\mathrm{C}}=\mathrm{O}$ were also observed in presence of the polyester. The peaks for the $\mathrm{PLA}_{50} / \mathrm{PVP}_{50}$ membranes were $[\underline{\mathrm{C}}-\mathrm{H} / \underline{\mathrm{C}}-\mathrm{C}] /[\underline{\mathrm{C}}-\mathrm{O}, \underline{\mathrm{C}}-\mathrm{N}] /[\underline{\mathrm{C}}=\mathrm{O}]=51 / 28 / 21$ and 53/27/20 for $\mathrm{PLA}_{50} / \mathrm{PVP}_{50} / \mathrm{Curc}_{15}$. For those two types, we concluded that the surface was enriched by fragments of the PLA macromolecules and to a lesser degree by the Curc (Curc $15 \mathrm{wt} \%$ ). As a final note, in the fibers containing Curc we also observed a peak at $291 \mathrm{eV}$, consistent with $\pi \rightarrow \pi^{*}$ satellite peak, characteristic for the benzene rings of Curc.

It is well known that PVP is a water soluble and biocompatible polymer easily used for the production of

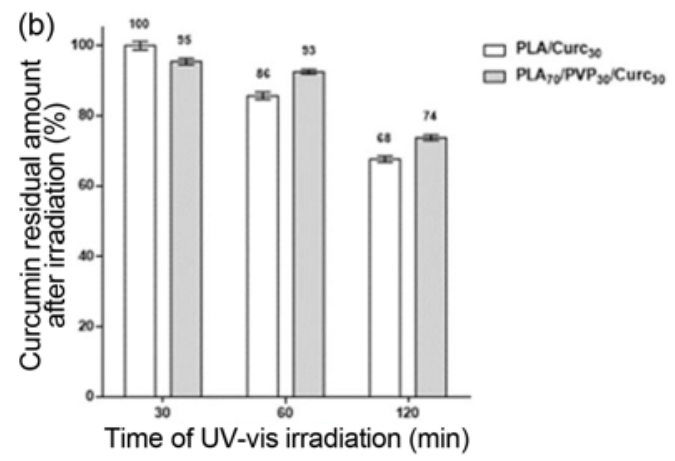

Figure 3. Curc residual amount in the PVP/Curc (a) and PLA/(PVP/)Curc (b) membranes, after 30, 60 and 120 min of irradiation with UVVis light source (wavelength range: from 200 to $600 \mathrm{~nm}$, samples source distance of $43 \mathrm{~cm}$ ). 


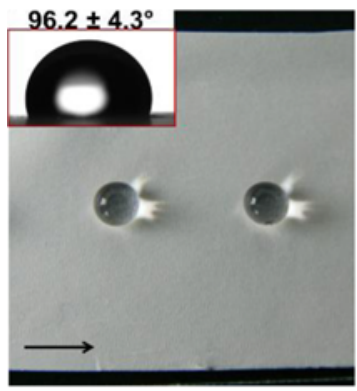

(a)

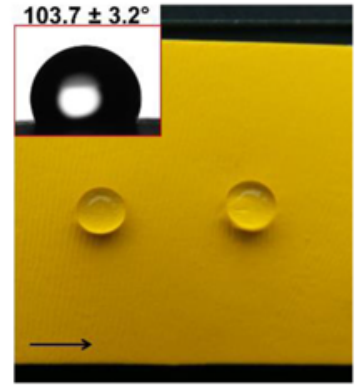

(b)

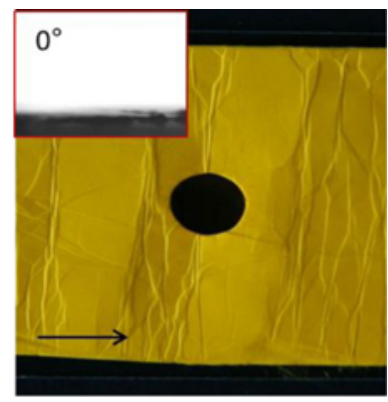

(c)

Figure 4. Digital images of $\mathrm{PLA}_{50} / \mathrm{PVP}_{50}$ (a), $\mathrm{PLA}_{50} / \mathrm{PVP}_{50} / \mathrm{Curc}_{15}$ (b) and $\mathrm{PVP} / \mathrm{Curc}_{25}$ (c) membrane with deposited water droplets. The collector rotation direction is indicated with an arrow.

hydrogels [36]. In cases of an open wound, with a rough surface and unequal depth, PVP/Curc membranes could cover the wound surface by forming a thin hydroscopic gellike layer under the influence of the exudate. In this way, the membranes will act as an active wound dressing and the locally delivered Curc and exercise its therapeutic properties. In this aspect the electrospun materials hydrophobic/hydrophilic behavior was evaluated performing water contact angle tests (deposition of distilled water drops onto the membranes surface). For the $\mathrm{PLA}_{50} / \mathrm{PVP}_{50}$ and $\mathrm{PLA}_{50} / \mathrm{PVP}_{50} / \mathrm{Curc}_{15}$ the values were respectively $96.2 \pm 4.3^{\circ}$ and $103.7 \pm 3.2^{\circ}$ (Figure 4). The higher hydrophobic character of the $\mathrm{PLA}_{50} / \mathrm{PVP}_{50} /$ Curc $_{15}$ membrane was explained with the presence of two hydrophobic components in the fibers - the biocompatible polyester and the polyphenol compound. As expected, the fibrous materials made only of PVP and PVP/Curc (water soluble polymer matrix), were characterized by a fast wetting process followed by a swelling leading to the loss of the membranes fibrous structure. The water contact angle values were a match for those from the XPS analysis, in regard of the membranes surface enrichment with hydrophobic/ hydrophilic fragments.

In support of the results so far were the data acquired upon immersion of the $\mathrm{PLA}_{50} / \mathrm{PVP}_{50}$ and $\mathrm{PLA}_{50} / \mathrm{PVP}_{50} / \mathrm{Curc}_{15}$ membranes for $24 \mathrm{~h}$ in aqueous solution (distilled water). It was established that the membranes undergo 50 and $57 \%$ of total weight loss respectively, explained by the diffusion of PVP and PVP/Curc (yellow coloring of the solution) from the fibers. Consequently, fibers morphological changes were observed: instead of the usual smooth surface with a cylindrical cross section, they assumed with a ribbon-like form until the formation of canals along their length. From the presented SEM micrographs, it was found that the membranes porous structure was preserved without additional fiber interconnection (Figure 5). Similar morphological changes in fibers made of hydrophobic and hydrophilic polymers and their blends, were described by others [37,38].

The morphological characteristics of the fibers are another parameter highly affecting the bioactive substance release

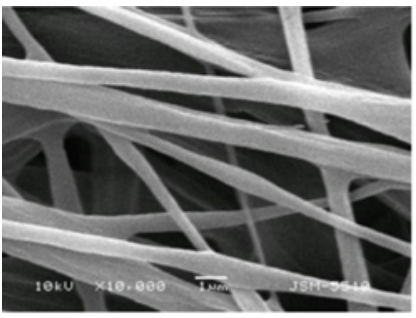

(a)

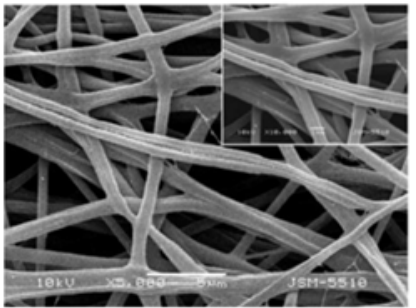

(c)

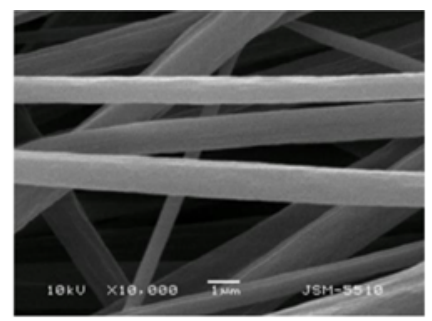

(b)

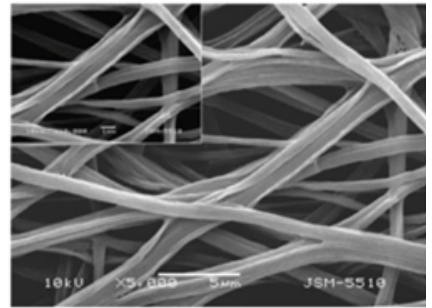

(d)
Figure 5. SEM micrographs of $\mathrm{PLA}_{50} / \mathrm{PVP}_{50}$ and $\mathrm{PLA}_{50} / \mathrm{PVP}_{50} /$ Curc $_{15}$ fibers - before ( $\mathrm{a}$ and $\mathrm{b}$ ) and after (c and d) $24 \mathrm{~h}$ immersion in distilled water. Magnification of $\times 10000$ for $(a$ and $b)$ and of $\times 5000(\times 10000$ for the insets $)$ for $(c$ and $d)$.

profile. Some are related to the presence of pores on the fibers surface, their mean diameter, design (monolithic or core-sheath type fibers), polymer matrix composition and its degree of reticulation, as well as the ability of the active agent to interact with the polymer material components. Over the last years, the scientific efforts in Curc delivery systems design were focused mainly on improving its water solubility. It has been demonstrated that when hydrophobic polymers [poly(butylene succinate), poly( $\varepsilon$-caprolactone)] are used, the quantity of the released lipophilic natural product is lower because of its significant affinity to the polymer matrix than the release medium $[39,40]$. In such cases, the diffusion of Curc from the fibers to the solution could be influenced by adding surfactants (Tween20, sodium dodecyl sulfate, cetrimonium bromide and others) or solvents (methanol, DMSO) to the buffer solution with 
desired $\mathrm{pH}$. Often, they form complex based on hydrogen bonds with the antioxidant thus increasing its water solubility [41,42]. Another interesting approach to influence release profile of Curc is the use of polymer matrix (polymers blends or copolymers) based on hydrophobic and biocompatible hydrophilic polymer e.g. PEG, PVA, oligosaccharides (cyclodextrin) [8,43]. These macromolecules influence not only the wetting of the membranes, but they also favor the diffusion of Curc from the fibers by creating water soluble complex. The subsequent diffusion of the complex leads to an increase in the material solvent contact surface, thus enhancing its degree of wetting. By the fabrication of core/sheath-type fibers, from hydrophobic and hydrophilic polymers, it is possible to attain desired degree of the membranes wetting and greater control over the active substances release profile [27,44].

In this study Curc release profile was studied on two types of membranes, $\mathrm{PLA}_{50} / \mathrm{PVP}_{50} / \mathrm{Curc}_{15}$ and PLA/Curc 30 . The fibers containing the water-soluble polymer showed fast first release stage for the natural product (Figure 6). This was explained by the initial process of wetting and swelling of the membranes, which was responsible for the Curc

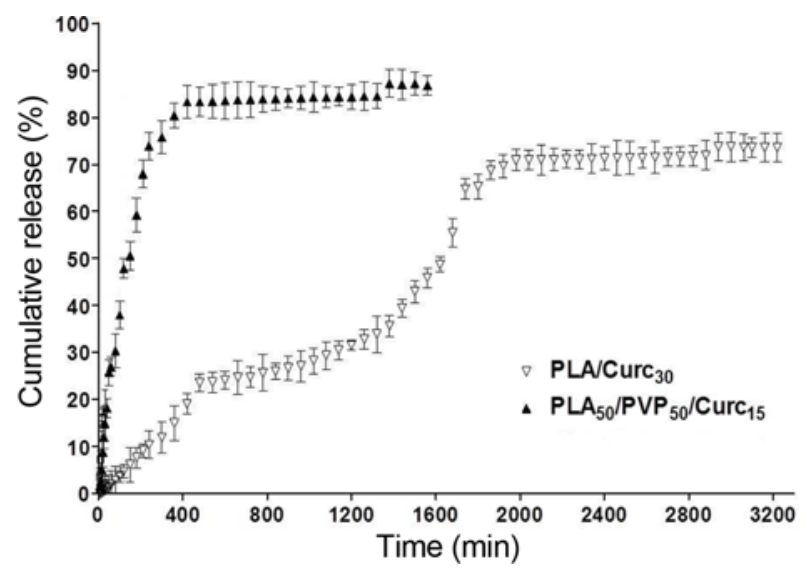

Figure 6. Cumulative release profile of Curc from PLA/Curc ${ }_{30}$ and $\mathrm{PLA}_{50} / \mathrm{PVP}_{50} / \mathrm{Curc}_{15}$ membranes (acetate buffer/PVP (90/10 $\mathrm{v} / \mathrm{v}), \mathrm{pH}$ of 5.5 and temperature of $37^{\circ} \mathrm{C}$ ).

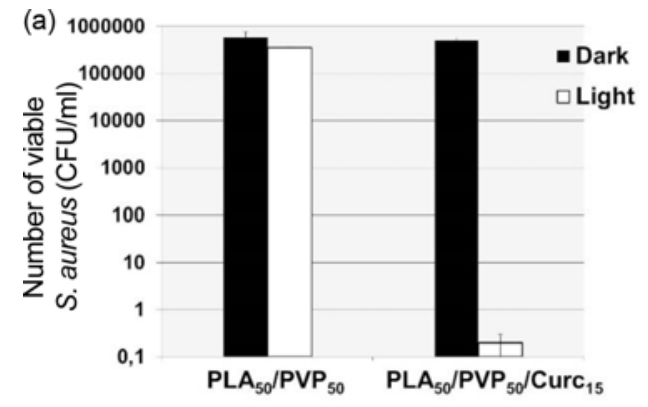

diffusion located mainly on the fibers surface. The second release stage was associated to a slower diffusion process of the active substance from the volume of the fibers. The amount of the released Curc for these fibers was up to $85 \%$ of its initial values in the membranes, thus rendering the materials suitable for the design of delivery systems with fast biological effect. For the hydrophobic PLA/Curc 30 fibers, the release profile was significantly slower with a first stage up to 400 min and accompanied with a much lower amount of the diffused polyphenol (25\%). This was explained by the enrichment of the fibers surface with C-H/ C-C chemical bonds, which makes their level of wetting significantly lower. The maximum amount of the released Curc was around $70 \%$ in this case. These results reveal that PVP facilitate Curc release.

Bacterial infections are a serious threat to overall health and could lead to sever complications and chronic clinical conditions. Often antibiotics are considered as the only cure leading to an increase in microorganisms' overall drug resistance. Curc is also known for its excellent antibacterial and antifungal properties, making it suitable candidate in term of antibiotic replacement. In this study we performed a series of microbiological experiments with the goal of evaluate the $\left.\mathrm{PLA}_{50} / \mathrm{PVP}_{50} / \mathrm{Curc}_{15}\right)$ fibers impact on pathogens growth inhibition. First, we determined the minimal inhibitory concentration of the natural product against both strains $S$. aureus and C. albicans strains: 15 and $2 \mu \mathrm{g} / \mathrm{ml}$, respectively. Then the membranes were put in contact with the bacterial suspension in dark environment or upon irradiation at $420 \mathrm{~nm}$. Only the membranes irradiated for $60 \mathrm{~min}$, demonstrated bactericidal effect after $24 \mathrm{~h}$ of the microbiological culture incubation (Figure 7). The lightdepended antibacterial properties were in accordance with our previous results on similar systems [11].

As part of its therapeutic properties, Curc is well known for its antitumor effect. In order to evaluate the potential application of the membranes as biomaterials, tests with HeLa and Graffi cancer cell lines (cells in cancer forms of cervical cancer and myeloid tumor) were performed. The kinetics of the cell viability were established via MTT test,

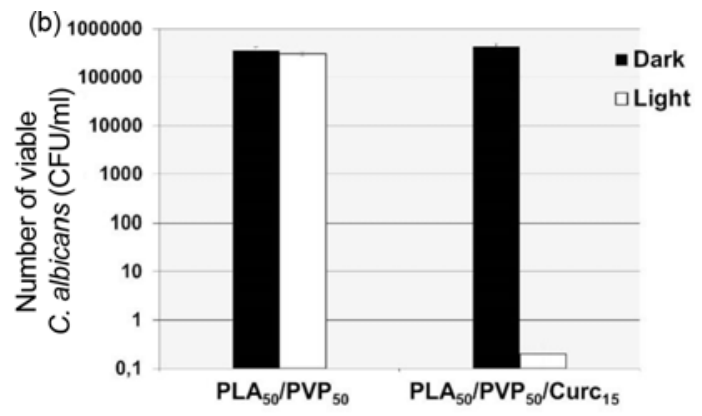

Figure 7. Antibacterial activity of $\mathrm{PLA}_{50} / \mathrm{PVP}_{50}\left(/ \mathrm{Curc}_{15}\right)$ membranes against $S$. aureus (a) and C. albicans (b). Incubation time of 30 min, with ( $\square$ ) or without $(\square)$ irradiation $(1 \mathrm{~h}, \lambda=420 \mathrm{~nm})$. 

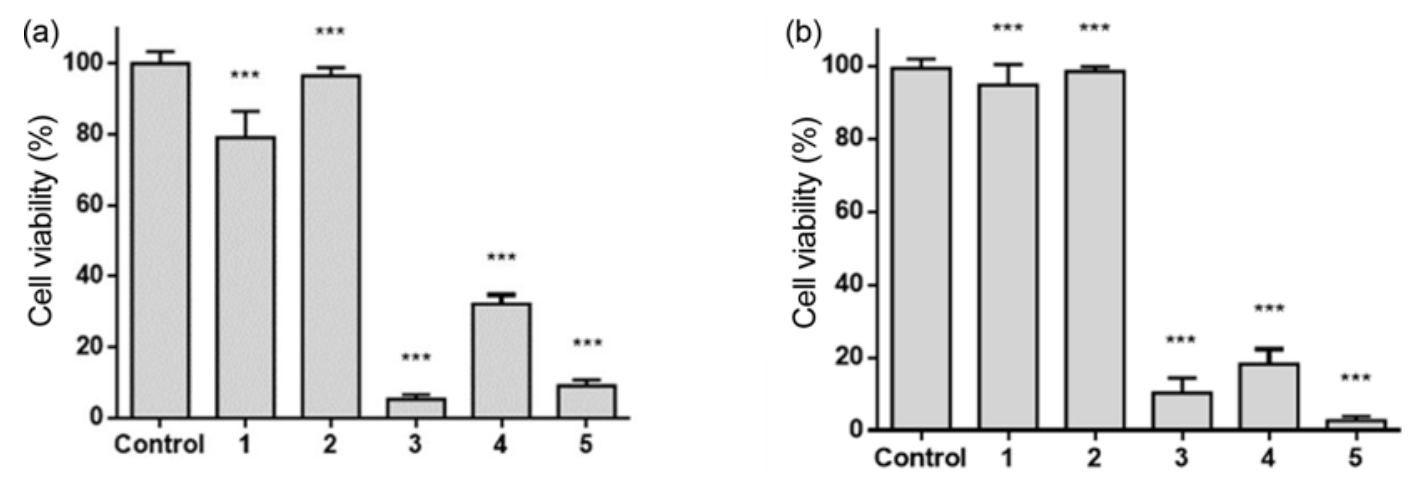

Figure 8. Cell viability of HeLa (a) and Graffi (b) cancer cell lines after $72 \mathrm{~h}$ contact with membranes of PLA $\mathrm{P}_{50} / \mathrm{PVP}_{50}(1), \mathrm{PVP}(2), \mathrm{PLA}_{50} /$ $\mathrm{PVP}_{50} /$ Curc $_{15}$ (Curc in the membrane - $\left.11 \mu \mathrm{g}\right)(3), \mathrm{PVP} /$ Curc $_{15}$ (Curc in the membrane $\left.11 \mu \mathrm{g}\right)(4)$ and Curc $(11 \mu \mathrm{g} / \mathrm{ml})(5)$.

at the 24, 48 and $72 \mathrm{~h}$ of cell suspension contact with the (PLA/)PVP/Curc membranes. From the data presented in Figure 8, it was found that membrane of $\mathrm{PLA}_{50} / \mathrm{PVP}_{50} /$ $\operatorname{Curc}_{15}$ (Curc $11 \mu \mathrm{g} / \mathrm{m} l$ ) had cytotoxic effect comparable to Curc at the same solution concentration $(11 \mu \mathrm{g} / \mathrm{m} l)$ : up to $95 \%$ suppression of cancer cell proliferation for the HeLa line and up to $85 \%$ for the Graffi line. As we already pointed out, the fiber polymer matrix composition is also having an impact on Curc diffusion. The longer release phase of the antioxidant from the $\mathrm{PLA}_{50} / \mathrm{PVP}_{50} / \mathrm{Curc}_{15}$ fibers was a premise for a persistent cytotoxic effect, in comparison to the PVP/Curc membrane where fast delivery is occurring accompanied with short biological effect. At the same time, the cancer cell viability in contact with the control PLA $\mathrm{P}_{50} / \mathrm{PVP}_{50}$ and PVP membranes was not affected and any additional level of cell proliferation was observed. The results were in agreement with the scientific literature regarding the specific cytotoxic effect against tumor cells of Curc loaded electrospun materials [12,21].

As a next step it was of interest to evaluate the immune response of normal cells in presence of the membranes. We developed a series of in vitro tests with mouse peritoneal macrophages and spleen lymphocytes. It was found out that the PVP and $\mathrm{PLA}_{50} / \mathrm{PVP}_{50}$ membranes were responsible for

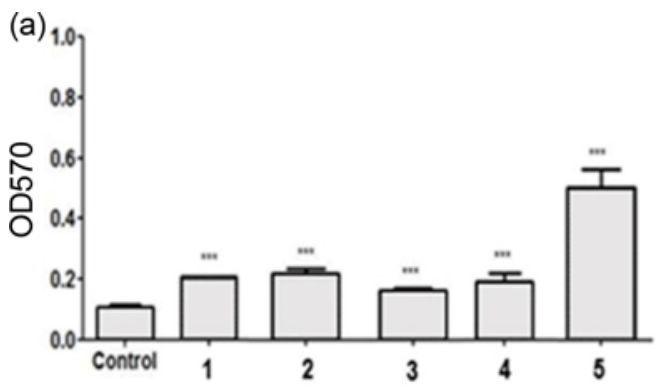

a slight microphages activation (Figure 9(a)), accompanied by an increased spleen lymphocytes cell division (Figure 9(b)). This was a proof of the activated immune response towards the materials. In the case of the $\mathrm{PLA}_{50} / \mathrm{PVP}_{50} / \mathrm{Curc}_{15}$ and $\mathrm{PVP} / \mathrm{Curc}_{15}$ membranes, a lessened activity in the immune response (reduced proliferation of white blood cells) was detected, indicating the improved membranes biocompatibility. The results were consistent with data in the scientific literature with regards of lymphocytes and macrophages cell proliferation $[45,46]$.

\section{Conclusion}

In the present contribution, we developed bioactive microfibrous PVP/Curc and PLA/PVP/Curc membranes by electrospinning. The spinning solution dynamic viscosity depended on the Curc amount with direct impact on the fibers morphology (average diameter and shape). As a result of the hydrogen bond-based complex formation between Curc and PVP macromolecules, Curc remained in the amorphous state in the membranes. It was proven that the amount, physico-chemical and therapeutic properties of curcumin were preserved upon UV-Vis irradiation, offering the possibility to UV light sterilize the fibrous biomaterials.

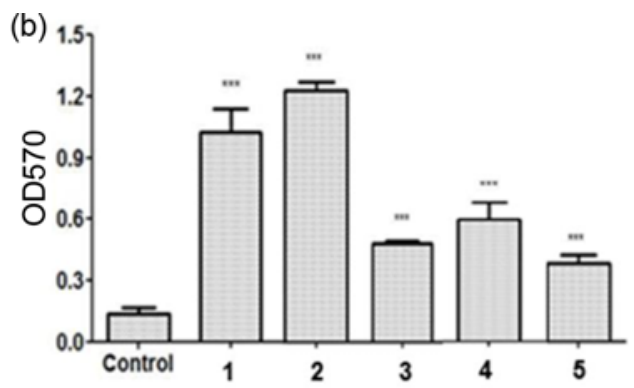

Figure 9. Cell proliferation of mouse peritoneal macrophages (a) and spleen lymphocytes (b) after $72 \mathrm{~h}$ of contact with the fibrous materials of $\mathrm{PLA}_{50} / \mathrm{PVP}_{50}(1), \mathrm{PVP}(2), \mathrm{PLA}_{50} / \mathrm{PVP}_{50} / \mathrm{Curc}_{15}$ (Curc in the membrane - $11 \mu \mathrm{g}$ ) (3), PVP/Curc 15 (Curc in the membrane - $\left.11 \mu \mathrm{g}\right)(4)$ and Curc $(11 \mu \mathrm{g} / \mathrm{m} l)(5)$. 
The curcumin release profile depended on the hydrophobic/ hydrophilic nature of the polymer matrix components, and the incorporation of PVP facilitated Curc release from the fibers. The microbiological studies revealed a clear bactericidal effect of the membranes against the Gram-positive bacteria $S$. aureus and the fungus $C$. albicans. It was proven that both $\mathrm{PVP} / \mathrm{Curc}$ and PLA/PVP/Curc membranes carried cytotoxic properties against $\mathrm{HeLa}$ and Graffi cancer cells. The immune response to normal cells $(72 \mathrm{~h}$ contact with murine peritoneal macrophages and spleen lymphocytes) showed an increased biocompatibility of the curcumin-loaded materials vs. the control group of blank PVP and PLA/PVP membranes. The electrospun (PLA/)PVP/Curc materials could find application as biomaterials in open wounds treatment, where antibacterial or antifungal properties are desired or as potential implants with antitumor properties.

\section{Acknowledgements}

The authors thank the National Science Fund of Bulgaria for the financial support (Grant DFNI-T02/1/12.12.2014).

Electronic Supplementary Material (ESM) The online version of this article (doi: 10.1007/s12221-020-9473-z) contains supplementary material, which is available to authorized users.

\section{References}

1. M. Ignatova, I. Rashkov, and N. Manolova, Expert Opin. Drug Del., 10, 469 (2013).

2. A. J. Hassiba, M. E. E. Zowalaty, G. K. Nasrallah, T. J. Webster, A. S. Luyt, A. M. Abdullah, and A. A. Elzatahry, Nanomedicine, 11, 715 (2016).

3. A. Toncheva, M. Spasova, D. Paneva, N. Manolova, and I. Rashkov, Int. J. Polym. Mater. Po., 63, 657 (2014).

4. I. Chattopadhyay, K. Biswas, U. Bandyopadhyay, and R. K. Banerjee, Curr. Sci., 87, 44 (2004).

5. B. T. Kurien, A. Singh, H. Matsumoto, and R. H. Scofield, AASSAY Drug Dev. Techn., 5, 567 (2007).

6. H. H. Tønnesen, M. Másson and T. Loftsson, Int. J. Pharm., 244, 127 (2002).

7. O. Naksuriya, S. Okonogi, R. M. Schiffelers, and W. E. Hennink, Biomaterials, 35, 3365 (2014).

8. G. Yakub, A. Toncheva, N. Manolova, I. Rashkov, V. Kussovski, and D. Danchev, J. Bioact. Compat. Pol., 29, 607 (2014).

9. G. Yakub, A. Toncheva, N. Manolova, I. Rashkov, D. Danchev, and V. Kussovski, J. Appl. Polym. Sci., 133, 1 (2016).

10. E. Thangaraju, S. Rajiv, and T. S. Natarajan, J. Polym. Res., 22, 1 (2015).

11. P. B. Tsekova, M. G. Spasova, N. E. Manolova, N. D. Markova, and I. B. Rashkov, Mater. Sci. Eng. C, 73, 206
(2017).

12. P. Tsekova, M. Spasova, N. Manolova, I. Rashkov, N. Markova, A. Georgieva, and R. Toshkova, J. Mater. Sci. Mater-M., 29, 1 (2017).

13. X.-Z. Sun, G. R. Williams, X.-X. Hou, and L.-M. Zhu, Carbohydr. Polym., 94, 147 (2013).

14. M. Ranjbar-Mohammadi and S. H. Bahrami, Int. J. Biol. Macromol., 84, 448 (2016).

15. B. Dhurai, N. Saraswathy, R. Maheswaran, P. Sethupathi, P. Vanitha, S. Vigneshwaran, and V. Rameshbabu, Front. Mater. Sci., 7, 350 (2013).

16. N. Killi, V. L. Paul, and R. V. N. Gundloori, New J. Chem., 39, 4464 (2015).

17. Z. Chen, Z. Chen, A. Zhang, J. Hu, X. Wang, and Z. Yang, Biomater. Sci., 4, 922 (2016).

18. E. Thangaraju, N. T. Srinivasan, R. Kumar, P. K. Sehgal, and S. Rajiv, Fiber. Polym., 13, 823 (2012).

19. C. Wang, C. Ma, Z. Wu, H. Liang, P. Yan, J. Song, N. Ma, and Q. Zhao, Nanoscale Res. Lett., 10, 2 (2015).

20. M. Sampath, R. Lakra, P. Korrapati, and B. Sengottuvelan, Colloid. Surface B, 117, 128 (2014).

21. G. Guo, S. Fu, L. Zhou, H. Liang, M. Fan, F. Luo, Z. Qian, and Y. Wei, Nanoscale, 3, 3825 (2011).

22. T. Mosmann, J. Immunol. Methods, 65, 55 (1983).

23. A. Koski, K. Yim, and S. Shivkumar, Mater. Lett., 58, 493 (2004).

24. D. Hussain, F. Loyal, A. Greiner, and J. H. Wendorff, Polymer, 51, 3989 (2010).

25. A. Paradkar, A. A. Ambike, B. K. Jadhav, and K. R. Mahadik, Int. J. Pharm., 271, 281 (2004).

26. S. L. Shenoy, W. D. Bates, H. L. Frisch, and G. E. Wnek, Polymer, 46, 3372 (2005).

27. R. Sedghi and A. Shaabani, Polymer, 101, 151 (2016).

28. A. Rahma, M. M. Munir, Khairurrijal, A. Prasetyo, V. Suendo, and H. Rachmawati, Biol. Pharm. Bull., 39, 163 (2016).

29. G. Zhang, J. Zhang, X. Zhou, and D. Shen, J. Appl. Polym. Sci., 88, 973 (2003).

30. M. Kakran, N. G. Sahoo, Y. W. Tan, and L. Li, Colloid. Surface, 433, 111 (2013).

31. M. Vert, P. Christel, F. Chabot, and J. Leray in "Macromolecular Biomaterials" (G. W. Hastings and P. Ducheyne Eds.), Chap.4, pp.119-142, CRC Press, Boca Raton, FL, 1984.

32. S. Li, J. Biomed. Mater. Res. B, 48B, 342 (1999).

33. T. Kongkhlang, K. Tashiro, M. Kotaki, and S. Chirachanchai, J. Am. Chem. Soc., 130, 15460 (2008).

34. C. R. A. Souza, S. F. Osme, and M. B. A. Gloria, J. Food Process. Pres., 21, 353 (1997).

35. K. M. Krasowska, J. Zawala, and K. Malysa, Adv. Colloid Interf., 147-148, 155 (2009).

36. D.-G. Yu, X.-X. Shen, C. Branford-White, K. White, L.-M. Zhu, and A. Bligh, Nanotechnology, 20, 1 (2009).

37. H. T. Bui, O. H. Chung, J. Dela Cruz, and J. S. Park, 
Macromol. Res., 22, 1288 (2014).

38. M. Bognitzki, T. Frese, M. Steinhart, A. Greiner, J. H. Wendorff, A. Schaper, and M. Hellwig, Polym. Eng. Sci., 41, 982 (2001).

39. E. Llorens, H. Ibañez, L. J. del Valle, and J. Puiggalí, Mater. Sci. Eng. C, 49, 472 (2015).

40. J. Shubham, M. Sai Rama Krishna, and C. Kaushik, Biomed. Mater., 11, 1 (2016).

41. L. Deng, X. Kang, Y. Liu, F. Feng, and H. Zhang, Food Chem., 231, 70 (2017).
42. R. Sedghi, A. Shaabani, Z. Mohammadi, F. Y. Samadia, and E. Isaei, Carbohydr. Polym., 159, 1 (2017).

43. W. Chen, H. EI-Hamshary, S. S. Al-Deyab, and X. Mo, Adv. Polym. Tech., 37, 647 (2018).

44. Z. Aytac and T. Uyar, Int. J. Pharm., 518, 177 (2017).

45. H. Yang, W. Xu, Z. Zhou, J. Liu, X. Li, L. Chen, J. Weng, and Z. Yu, Exp. Clin. Endocrinol. Diabetes, 123, 360 (2015).

46. S. Antony, R. Kuttan, and G. Kuttan, Immunol. Invest., 28, 291 (1999). 